\title{
A rigid sphere approach to positron elastic scattering by noble gases, molecular hydrogen, nitrogen and methane
}

\author{
Kamil Fedus ${ }^{\mathrm{a}}$ \\ Institute of Physics, Faculty of Physics, Astronomy and Informatics, Nicolaus Copernicus University, Grudziadzka 5/7, \\ 87-100 Toruń, Poland
}

Received 18 July 2016 / Received in final form 10 October 2016

Published online 13 December 2016

(C) The Author(s) 2016. This article is published with open access at Springerlink.com

\begin{abstract}
A simple potential model of a rigid sphere combined with an adiabatic dipole polarization $\left(\sim r^{-4}\right)$ is tested for positron-atom and positron-molecule elastic collisions. The numerical model, which is based on the analytical solution of radial Schrödinger equation for $r^{-4}$ potential, depends solely upon the average dipole polarizability of the target and one adjustable parameter - the radius of a hard core. The validity of model is assessed by an extensive comparative study against numerous experimental cross-sections and theoretical phase-shifts of angular momentum partial waves for positrons scattered elastically by $\mathrm{He}$, Ne, $\mathrm{Ar}, \mathrm{Kr}, \mathrm{Xe}, \mathrm{H}_{2}, \mathrm{~N}_{2}$ and $\mathrm{CH}_{4}$. In particular it is shown that this very simple approach can be used to model positron elastic collisions with targets characterized by moderate dipole polarizabilities $\left(\mathrm{Ar}, \mathrm{Kr}, \mathrm{H}_{2}, \mathrm{~N}_{2}\right)$ in good agreement with experiments for impact energies covering almost entire range from the positronium formation threshold down to the zero energy.
\end{abstract}

\section{Introduction}

The recent progress in experimental studies on low-energy positron scattering by noble gases greatly improved our understanding of positron binary interactions with atomic targets for impact energies below the positronium formation threshold (see review papers $[1,2]$ ). In particular, the energy dependencies of integral elastic scattering crosssections for helium ( $\mathrm{He}$ ) and neon $(\mathrm{Ne})$ were confirmed to be characterized by valley-like shapes, which are similar to narrow Ramsauer-Townsend minima observed in electron scattering from heavier noble gasses: argon (Ar), krypton $(\mathrm{Kr})$ and xenon $(\mathrm{Xe})$. On the other hand the scattering cross-sections for positron collisions with the last three atoms exhibit completely different shapes when compared to He and Ne: the steep decrease of magnitude with increasing energy ended by almost flat (virtually independent of energy) part just below the positronium formation threshold. Interestingly, the similar shapes have been also found for small nonpolar molecular targets such as hydrogen $\left(\mathrm{H}_{2}\right)$, nitrogen $\left(\mathrm{N}_{2}\right)$ and methane $\left(\mathrm{CH}_{4}\right)$ (see recent papers [3-5] and references therein). These similarities indicate that interaction potentials between positron and different targets are characterized by some target-independent features. In addition, Karwasz [7-9] noticed that averaged magnitudes of "flat" part of scattering cross-sections scale in a similar way as atomic and molecular dimensions. This effect is similar to classical elastic scattering on hard sphere. Recently, in more detailed study [10] we showed that the atomic radii de-

\footnotetext{
${ }^{a}$ e-mail: kamil@fizyka.umk.pl
}

termined from positron scattering data using a classical rigid sphere model are comparable to covalent radii of atoms. However this simple picture does not translate directly into the quantum mechanics since pure quantummechanical hard-sphere model is not applicable to lowenergy positron scattering [11].

From a theoretical point of view the scattering of low-energy small charged particles (such as electron and positron) from atoms and molecules in a gas phase is a very attractive research field allowing for validation of different quantum scattering models. On one hand, many theoretical models were proved to be able to describe a delicate counterbalance between attractive short-range static - polarization and repulsive exchange interactions present in low-energy electron elastic scattering. On the other hand, the theoretical description of positron elastic collisions was found to be much more challenging, particularly for ab initio procedures, despite the fact that no exchange interaction is present (for example see Ref. [6] and references therein). For positrons the repulsive static potential is counterbalanced solely by an attractive polarization potential making computational approaches very sensitive to the short-range positron - target's electrons (nonadiabatic) correlations. High quality (but computationally expensive) models have been developed to challenge this problem including, for example, approximate density functional theory (DFT) by Gianturco et al. [12], polarizedorbital calculations by McEachran and Stauffer [13] and McEachran et al. [14-18], many-body theory with virtual positronium formation by Green et al. [19], convergent close-coupling (CCC) method by Fursa and Bray [20] 
and semi-empirical potential model for ab initio calculations by Assafrão et al. [21] and Poveda et al. [22]. All these approaches were able to successfully reproduce shapes and magnitudes of experimental elastic scattering cross-sections for most noble gases, though they are not fully consistent, particularly in the prediction of scattering lengths. For molecular targets the situation is less optimistic since different diverging results were published for the same molecules. This is also true for the simplest target, $\mathrm{H}_{2}$ (see Ref. [3] and references therein).

Given these difficulties related to ab initio approaches, the semiempirical models gain in value since they have potential to capture main properties of positron interaction with atomic and molecular targets with relatively small computational costs. Schrader [23] suggested that the most important feature of any semiempirical model for positron-atom elastic collisions is to have a correct longrange behavior, that is an adiabatic polarization potential proportional to $r^{-4}$. Using this potential, one can propose any approach with few disposable parameters to approximate unknown short-range nonadiabatic correlation interaction. In particular Schrader [23] proposed a simple model with only one adjustable parameter. More recently, in a series of papers [24-29] we showed that the low-energy electron and positron scattering by noble gases and simple non-polar molecules (such as $\mathrm{H}_{2}, \mathrm{CH}_{4}$ ) can be very well modeled using well-known modified effective range theory (MERT). In MERT the scattering problem is solved analytically considering the target as single object interacting with the incoming projectile through the long-range dipole polarization potential $\left(\sim r^{-4}\right)$. The contribution of shortrange many body interactions is included within the frame of this model as an energy-dependent term. This term is described by four to six parameters that are empirically found by comparison with experiment.

In this work, combining the idea of Karwasz $[7,8]$ that positrons probe atomic dimensions as in classical collisions with hard spheres and the statement of Schrader [23] that any semiempirical approach with correct long range potential works for positron scattering, we propose the model of rigid sphere combined with long-range adiabatic dipole polarization $\left(\sim r^{-4}\right)$ (see Fig. 1). Though such potential does not reproduce the full behavior of positron-target interaction, we show that this model is a good approximation to describe the elastic scattering cross-sections for positron collisions with noble gases and small non-polar molecules $\left(\mathrm{H}_{2}, \mathrm{~N}_{2}\right.$ and $\left.\mathrm{CH}_{4}\right)$ over wide energy ranges. In particular the model is found to work well for targets with moderate polarizabilities where no Ramsauer-Townsend minimum is observed in integral cross-sections. Similar to the model of Schrader [23], the present approach requires only one adjustable parameter - the radius of rigid sphere. The latter is found to be compatible with the position of principal maximum in the radial distribution of outermost orbital (defined here as the atomic radius) for atomic targets. Providing the present model we benefit also from the fact that the de Broglie wavelength of positron with kinetic energy below the positronium formation threshold is usually comparable or much greater than the di-

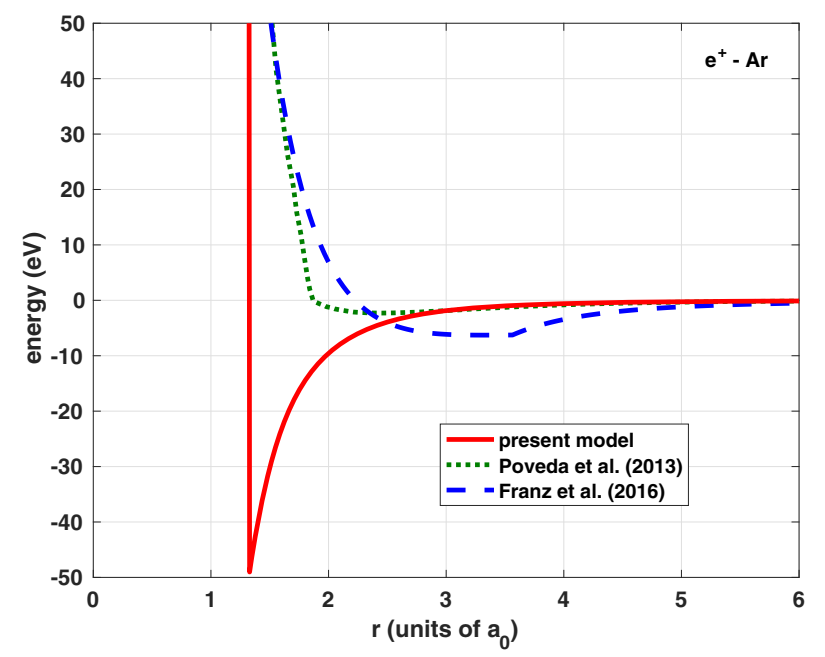

Fig. 1. Presently tested potential model shown for $e^{+}-\mathrm{Ar}$ collision. For comparison the potential energies used by Poveda et al. [22] and Franz et al. [10] are also presented.

mensions of small atoms and molecules. In such a case the interaction of low-energy charged particle with atomic and molecular targets tends to be governed by asymptotic long-range potentials and so, it is less sensitive to the details of target's internal structure, except cases when the resonance phenomena are present.

\section{Theoretical model}

We consider the following radial Schrödinger equation describing the relative motion of a charged particle and a non-polar spherical target (in atomic units):

$$
\left[\frac{\mathrm{d}^{2}}{\mathrm{~d} r^{2}}-\frac{l(l+1)}{r^{2}}-V(r)+k^{2}\right] \Psi_{l}(r)=0,
$$

where

$$
V(r)= \begin{cases}\infty, & r<R_{0} \\ -\alpha / r^{4}, & r>R_{0}\end{cases}
$$

with $R_{0}$ denoting the radius of rigid sphere and $\alpha$ being the static dipole polarizability. Here $\Psi_{l}(r)$ denotes the radial wave function for the partial wave $l$ and $k$ is the particle wavenumber.

The Schrödinger equation for the potential $V(r)$ and partial-wave quantum number $l$ can be solved exactly:

$$
\Psi_{l}(r)=\left\{\begin{array}{lr}
0, & r<R_{0} \\
C_{l} \sqrt{r} M_{v_{l}}(z)+\sqrt{r} T_{v_{l}}(z), & r>R_{0}
\end{array}\right.
$$

where $z=\ln (\sqrt{R} / \sqrt{k} r)$ with $R=\sqrt{\alpha e^{2} \mu / \hbar^{2}}$ being the characteristic distance - a typical length scale related to the $r^{-4}$ interaction. Here $e$ is the elementary charge, $\mu$ is the reduced mass of positron - target system and $\hbar$ is the Planck constant. Functions $M_{v_{l}}(z)$ and $T_{v_{l}}(z)$ are the two 
linearly independent solutions for $r^{-4}$ potential, so-called Mathieu functions [24]:

$$
\begin{aligned}
M_{v_{l}}(z) & =\sum_{n=-\infty}^{n=+\infty}(-1)^{n} c_{n} J_{2 n+v_{l}}(2 \sqrt{k R} \cosh z), \\
T_{v_{l}}(z) & =\sum_{n=-\infty}^{n=+\infty}(-1)^{n} c_{n} Y_{2 n+v_{l}}(2 \sqrt{k R} \cosh z),
\end{aligned}
$$

where $J(x)$ and $Y(x)$ are ordinary Bessel and Neumann functions. Coefficients $v_{l}(k)$ (Mathieu characteristic exponent) and $c_{n}(k)$ can be determined numerically from analytical properties of Mathieu functions, see appendices of references [24,25] where numerical procedures are described in details. Energy - dependent parameter $C_{l}(k)$ in equation (3) is determined from the continuity conditions for $\Psi_{l}(r)$ at $r=R_{0}$.

The asymptotic formulas for $M_{v_{l}}(z)$ and $T_{v_{l}}(z)$ for large and negative argument $z$ (large $r$ ) are following [24]

$$
\begin{aligned}
& M_{v_{l}}(z) \stackrel{z \rightarrow-\infty}{\longrightarrow} \sqrt{\frac{2}{\pi}} \frac{e^{z / 2}}{(k R)^{1 / 4}} m_{l} s_{v_{l}} \\
& \times \cos \left(\sqrt{k R} e^{-z}+\frac{\pi}{2} v_{l}-\frac{\pi}{4}\right), \\
& T_{v_{l}}(z) \stackrel{z \rightarrow-\infty}{\longrightarrow} \sqrt{\frac{2}{\pi}} \frac{e^{z / 2}}{(k R)^{1 / 4}} \frac{s_{v_{l}}}{m_{l}} \\
& \times\left[\sin \left(\sqrt{k R} e^{-z}+\frac{\pi}{2} v_{l}-\frac{\pi}{4}\right)\right. \\
&\left.-\left(m_{l}^{2}-1\right) \cot \left(\pi v_{l}\right) \cos \left(\sqrt{k R} e^{-z}+\frac{\pi}{2} v_{l}-\frac{\pi}{4}\right)\right],
\end{aligned}
$$

where $m_{l}(k)=\lim _{z \rightarrow 0^{+}} M_{v_{l}}(z) / M_{-v_{l}}(z)$ and $s_{v_{l}}(k)=$ $\sum_{n=-\infty}^{\infty} c_{n}(k)$.

Comparing asymptotic expression of present wavefunction with the standard asymptotic form of the scattered radial wave function, $\Psi_{l} \stackrel{z \rightarrow \infty}{\longrightarrow} G_{l} \sin (k r-l \pi / 2) / k r-$ $H_{l} \cos (k r-l \pi / 2) / k r$ one can easily derive an exact formula for the scattering phase-shift of $l$ th partial wave from $\tan \eta_{l}=-H_{l} / G_{l}$ :

$$
\tan \eta_{l}=\frac{\sin \delta_{l}-\left[C_{l} m_{l}^{2}+\cot \left(\pi v_{l}\right)\left(m_{l}^{2}-1\right)\right] \cos \delta_{l}}{\cos \delta_{l}+\left[C_{l} m_{l}^{2}+\cot \left(\pi v_{l}\right)\left(m_{l}^{2}-1\right)\right] \sin \delta_{l}},
$$

where $\delta_{l}=2 v_{l} / \pi+l+1 / 2$.

The exact formula can be used to calculate $\eta_{l}$ for all partial waves, however in order to speed up the computations one can use a simpler expression

$$
\tan \eta_{l} \approx \frac{\pi \alpha k^{2}}{8(l-1 / 2)(l+1 / 2)(l+3 / 2)}, \text { for } l>6
$$

because the difference between the latter formula and the exact results is insignificant for high $l$ for all considered targets.

The integral elastic scattering cross-section is calculated from standard partial wave expansion:

$$
\sigma=\frac{4 \pi}{k^{2}} \sum_{l=0}^{\infty}(2 l+1) \sin ^{2} \eta_{l}(k) .
$$

The only adjustable parameter in present model is $R_{0}$, the radius of the rigid sphere. In addition, the value of dipole polarizability $(\alpha)$ is needed but this quantity is generally well known for all studied targets because it has been determined accurately by different experimental and theoretical methods [30]. Moreover it was found that present calculations are very weakly sensitive to small changes of $\alpha$. On the contrary the model is very sensitive to the changes of hard sphere radius.

\section{Results}

The geometrical parameter $R_{0}$ has been optimized with trial and error method to obtain as good agreement as possible with experimental total cross-sections $(\sigma)$ and chosen theoretical scattering phase-shifts $\left(\eta_{l}\right)$. The standard values of static dipole polarizabilities were used [30]. All values of $R_{0}$ and $\alpha$ used in present calculations are listed in Table 1. For most targets the results of calculations for two or three different values of $R_{0}$ are given in order to illustrate how the output of present calculations changes with the size of rigid sphere.

For comparison purposes, Table 1 contains also the positions of principal maxima in the radial distributions of outermost orbitals for atomic targets as given by different sources [31-34]. Additionally, some estimates of sizes for molecular targets are also included in Table 1. For atoms, it is clear that values of $R_{0}$ used in present model are compatible with atomic radii defined in this way or at least they corresponds to typical radial positions of valence shells. In more details, for $\mathrm{He}$ and Ar, presently used hard core sizes match perfectly atomic radii reported in the literature. For $\mathrm{Kr}, R_{0}$ value falls in between positions of maxima of two occupied valence orbitals ( $4 s$ and $4 p$ ) as reported by Mann [34] (and similarly by Waber et al. [31]): $R_{4 s}=1.37 a_{0}$ and $R_{4 p}=1.56 a_{0}$. For Xe, the rigid sphere radii are closer to $R_{5 s}=1.71 a_{0}$ of $5 \mathrm{~s}$ orbital rather than the outermost one, $5 p: R_{5 p}=1.94 a_{0}$ as reported by Mann [34]. In the case of Ne, unlike for other atoms, the good agreement with scattering data was found using $R_{0}$ that is slightly higher than atomic radii given in all cited literature. Note however that above comparison has only a tentative character due to ambiguous definition of atomic radius in quantum physics. Nevertheless a relatively good correspondence between presently used rigid sphere radii and the literature data for atomic sizes confirms that the distortion of valence electrons cloud plays the most important role in the positron-atom collisions, whereas the interaction with inner-shell electrons is hindered by a strong repulsion with nucleus (the positron does not penetrate far inside the atom).

It is more difficult to define equivalent "molecular" radii for $\mathrm{H}_{2}, \mathrm{~N}_{2}$ and $\mathrm{CH}_{4}$ due to the lack of typical spherical symetry, though it has been proved [24-29] that approximation of spherical dipole polarizability is sufficient to describe low-energy positron (or electron) scattering by these molecules. In Table 1 we give different theoretical estimates of molecular sizes in order to show that values of $R_{0}$ used in present model are compatible with a typical molecular length-scale. Usually, the sizes of homonuclear 
Table 1. Static dipole polarizabilities $(\alpha)$ and radii of rigid sphere $\left(R_{0}\right)$ used in present calculations. For some targets more than one value of radius is used. For comparison the atomic radii defined as the principal maxima in the radial distributions of outermost orbitals and some theoretical estimates of molecular sizes are also given.

\begin{tabular}{|c|c|c|c|c|c|c|c|c|}
\hline \multicolumn{5}{|c|}{ Atoms } & \multicolumn{4}{|c|}{ Atomic radius $\left(a_{0}\right)$} \\
\hline Target & $\alpha\left(a_{0}^{3}\right)$ & \multicolumn{3}{|c|}{$R_{0}\left(a_{0}\right)$} & Waber and Cromer [31] & Fraga et al. [32] & Ghosh and Biswas [33] & Mann [34] \\
\hline $\mathrm{He}$ & 1.383 & 0.5762 & 0.588 & 0.6092 & 0.55 & 0.57 & 0.59 & 0.57 \\
\hline $\mathrm{Ne}$ & 2.671 & \multicolumn{3}{|c|}{0.8498} & 0.67 & 0.70 & 0.68 & 0.68 \\
\hline $\mathrm{Ar}$ & 11.23 & \multicolumn{3}{|c|}{1.3287} & 1.24 & 1.27 & 1.33 & 1.30 \\
\hline $\mathrm{Kr}$ & 16.86 & \multicolumn{2}{|c|}{1.4751} & 1.4977 & 1.50 & 1.61 & 1.79 & 1.56 \\
\hline $\mathrm{Xe}$ & 27.29 & \multicolumn{2}{|c|}{1.687} & 1.7239 & 1.86 & 1.89 & 2.42 & 1.94 \\
\hline \multicolumn{5}{|c|}{ Molecules } & \multicolumn{4}{|c|}{ Molecular sizes $\left(a_{0}\right)$} \\
\hline Target & $\alpha\left(a_{0}^{3}\right)$ & \multicolumn{3}{|c|}{$R_{0}\left(a_{0}\right)$} & \multicolumn{2}{|c|}{ Promolecule radii [35] } & \multicolumn{2}{|c|}{ Covalent radii } \\
\hline $\mathrm{H}_{2}$ & 5.314 & 0.9221 & 0.9509 & 0.9912 & \multicolumn{2}{|c|}{$1.04 \pm 0.38$} & \multicolumn{2}{|c|}{$0.59[36] ; 0.70[37]$} \\
\hline $\mathrm{N}_{2}$ & 11.54 & 1.2875 & 1.3249 & 1.4268 & \multicolumn{2}{|c|}{$1.45 \pm 0.25$} & \multicolumn{2}{|c|}{$1.02[38]$} \\
\hline $\mathrm{CH}_{4}$ & 16.52 & \multicolumn{2}{|c|}{1.4632} & 1.5445 & \multicolumn{4}{|c|}{ Carbon bonding radius: $1.36 a_{0}-1.47 a_{0}$ (see text for discussion) } \\
\hline
\end{tabular}

molecules such as $\mathrm{H}_{2}$ and $\mathrm{N}_{2}$ are described in terms of covalent radius, that is the half of bond length (internuclear distance). However, the $\mathrm{H}_{2}$ single-bond and the $\mathrm{N}_{2}$ triple-bond covalent radii are shorter than presently used values of $R_{0}$. An alternative definition of atomic radii in molecular diatomic systems is provided by a model of promolecule [35]. In this model the electron density distributions of each of atom in molecule is spherically averaged and placed at their minimum energy positions. The promolecule radius of diatomic homonuclear molecule is then defined to be the distance between the nucleus of the atom and the point of minimum electron density along the line between the nuclei [35]. Such definition of "molecular radius" yields mean results [35] which are more compatible with the sizes of hard spheres used in present model.

In heteronuclear $\mathrm{CH}_{4}$ molecule the $\mathrm{C}-\mathrm{H}$ internuclear separation ( $s p^{3}$ hybridisation) is around $2.06 a_{0}$ [39]. Considering the single-bond covalent radius of hydrogen to be between $0.59 a_{0}$ [36] and $0.7 a_{0}$ [37], the bonding radius of carbon atom in $\mathrm{CH}_{4}$ molecule falls in between $1.36 a_{0}$ and $1.47 a_{0}$. The latter values are comparable with present rigid sphere radii and with some estimates of carbon single-bond $(\mathrm{C}-\mathrm{C})$ covalent radius [36]. This coincidence may suggest that positron interacts rather with the carbon than hydrogen during the collision with methane molecule.

Please note that in order to avoid an overload of data presented in figures, in the following subsections the present calculations are compared only with selectively chosen experimental and theoretical results. Detailed comparison of high quality theories and experiments not included in the present work can be easily found elsewhere (for example see Ref. [2] and references therein).

\subsection{Helium}

Figure 2 shows a comparison of present calculations using three different values of $R_{0}$ with chosen experimental total cross-sections (TCS) and theoretical integral elastic cross-sections for positron collisions with helium. It is expected that the present model should be only a very rough

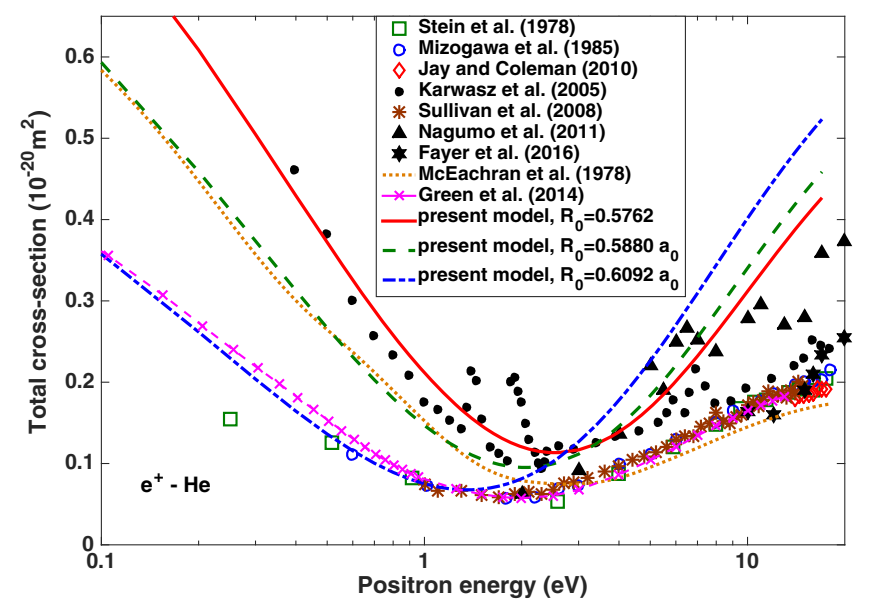

Fig. 2. Elastic scattering cross section for helium. Present calculations for three different rigid sphere radii $\left(R_{0}\right)$ are compared with chosen theoretical and experimental results. Theories: polarized orbital by McEachran et al. [14,15] and many-body theory by Green et al. [19]. Experiments: Stein et al. [40], Mizogawa et al. [41], Karwasz et al. [42-45], Sullivan et al. [46], Jay and Coleman [47], Nagumo et al. [48,49] and Fayer et al. [50].

approximation for description of $\mathrm{e}^{+}-$He collisions since the dipole polarizability of this target is too weak to approximate a correlation of two electrons with one positron by a simple adiabatic polarization potential. Moreover the static repulsion is probably not sufficiently strong to model it by infinite potential barrier at short range. Nevertheless the present approach with $R_{0}=0.5762 a_{0}$ is in quite good agreement (except the resonance structures) with experimental data by Karwasz et al. [51] at the low energy side and with data by Nagumo et al. [48,49] measured in magnetic field-free conditions at the high energy side. The latter dataset is supposed to be practically free from forward angle scattering error - the effect that tends to underestimate measured total cross-sections in attenuation-type experiments [52]. This can possibly explain why the TCS by Nagumo are higher than other data measured in presence of magnetic field in the scattering 
region. However the latest experiment by Fayer et al. [50] with another magnetic field-free system does not confirm the results of Nagumo et al. [48,49].

As shown in Figure 2 the increase of rigid sphere radius to $R_{0}=0.5880 a_{0}$ yields results that follows very closely polarized orbital calculations by McEachran et al. $[14,15]$ in low energy region $(<1 \mathrm{eV})$. Further increase of $R_{0}$ to $0.6092 a_{0}$ improves the agreement with experimental TCSs obtained by most experimental groups at very low energies $(<1 \mathrm{eV})$ while significantly enlarge the divergence between the model and experiments at higher energies. Noteworthy is the fact that the use of latter radius allows to reproduce (not exactly but with high degree of agreement) at low energies many-body theory by Green et al. [19]. Actually, we checked that it is possible to get good agreement at low energies $(<1 \mathrm{eV})$ with other theories, for example with close coupling calculations (CCC) by Wu et al. [53] (not shown in Fig. 2), by adjusting the radius of hard sphere in present model. It looks like a small shift in the position of the infinitive barrier yields similar effects (at least at very low energies) as using different theoretical approaches aiming to approximate nonadiabatic short-range correlation between positron and target's electrons. The similar observation holds also for other atomic and molecular targets.

\subsection{Neon}

Figure 3a shows the results of present calculations of elastic scattering cross-sections for positron collisions with neon. The good agreement with experimental data was found for $R_{0} \approx 0.85 a_{0}$. At low impact energies, $E<3 \mathrm{eV}$, present model agrees pretty well with available experimental TCSs. On the other hand, at higher energies it closely follows only the dataset by Nagumo et al. [56] measured in magnetic field-free conditions. The latter TCS, as for helium, is higher in magnitude than other measurements. The presently calculated scattering phase-shifts shown in Figure 3b for $s$-, $p$ - and $d$-partial waves resemble in shapes the results published by McEachran et al. [16] and Green et al. [19]. The important difference in magnitude appears only at higher energies, $E>3 \mathrm{eV}$, where the present model gives stronger negative $s$-wave and positive $p$-wave phase-shifts. This is reflected in higher integral cross-sections at higher energies when compared to both reference models, though the disagreement is much lower than for helium. Moreover, as for helium, we checked that it is possible to adjust $R_{0}$ in a way allowing to get much better agreement with polarized orbital [13-18] and many-body calculations [19] below $1 \mathrm{eV}$.

\subsection{Argon}

Figure $4 \mathrm{a}$ shows the results of present calculations (with $R_{0}=1.3287 a_{0}$ ) of elastic scattering cross-sections for positron collision with argon. The hard core radius has been chosen to give cross-section curve laying in between theoretical results by McEachran et al. [17] and Green et al. [19] in low energy limit. Note also (see Tab. 1) that
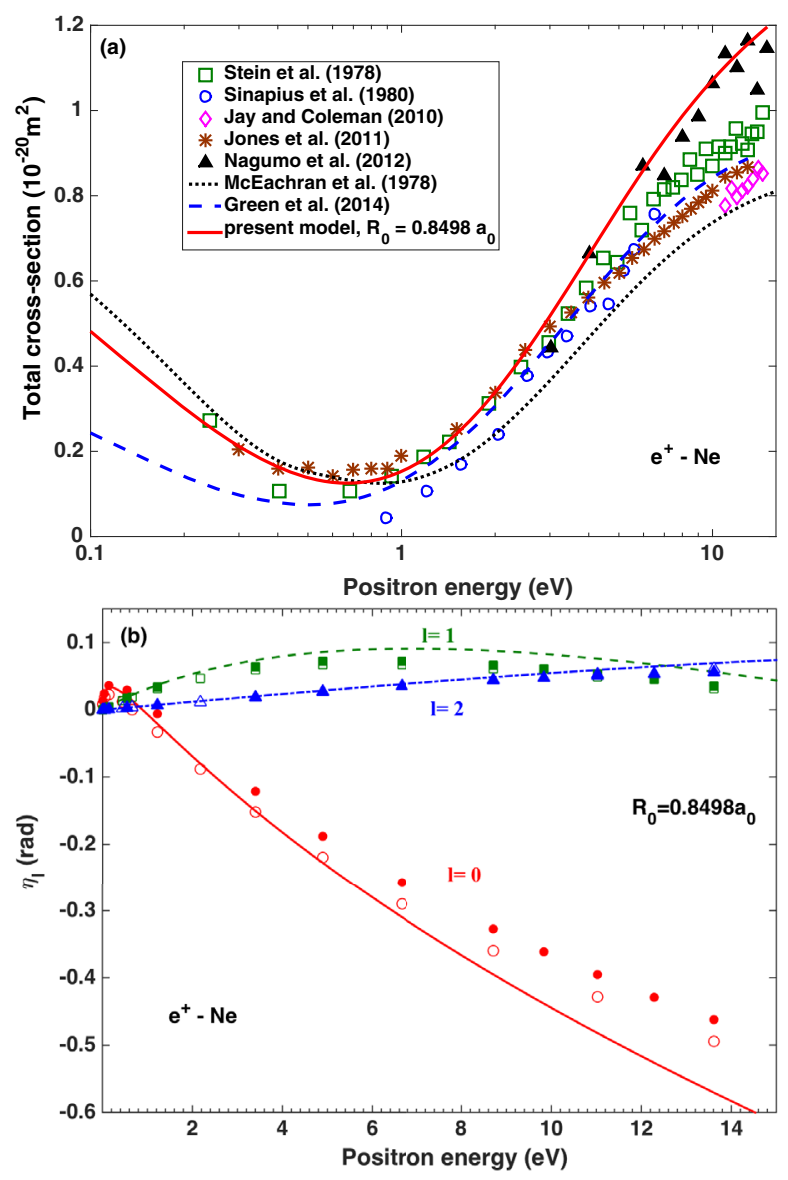

Fig. 3. (a) Elastic scattering cross section for neon. Present calculations are compared with chosen theoretical and experimental results. Theories: polarized orbital by McEachran et al. [16] and many-body theory by Green et al. [19]. Experiments: Stein et al. [40], Sinapius et al. [54], Jay and Coleman [47], Jones et al. [55], Nagumo et al. [56]. (b) Present $s-, p$ - and $d$-wave scattering phase-shifts $\left(\eta_{l}\right)$ compared with McEachran et al. [16] (filled symbols) and Green et al. [19] (empty symbols).

this radius is in very good agreement with the positions of principal maximum in radial distribution of argon outermost orbital calculated by different groups. The agreement with available experimental data has to be judged as excellent. Moreover present phase-shifts shown in Figure 4b follow closely the results reported by both reference theories. Similar to helium and neon the differences in phaseshift magnitudes between present model and both reference datasets appear only at higher energies, $E>6 \mathrm{eV}$, where the present model gives slightly stronger negative $s$-wave phase-shifts, but it does not translate into a significant difference in elastic integral cross-sections as observed for lighter atoms. Furthermore it is also possible to get cross-sections that reproduce both models in almost entire energy range below the positronium formation threshold by slightly adjusting $R_{0}$ (the changes are of the order of hundredths of the Bohr radius when compared to value quoted above). 

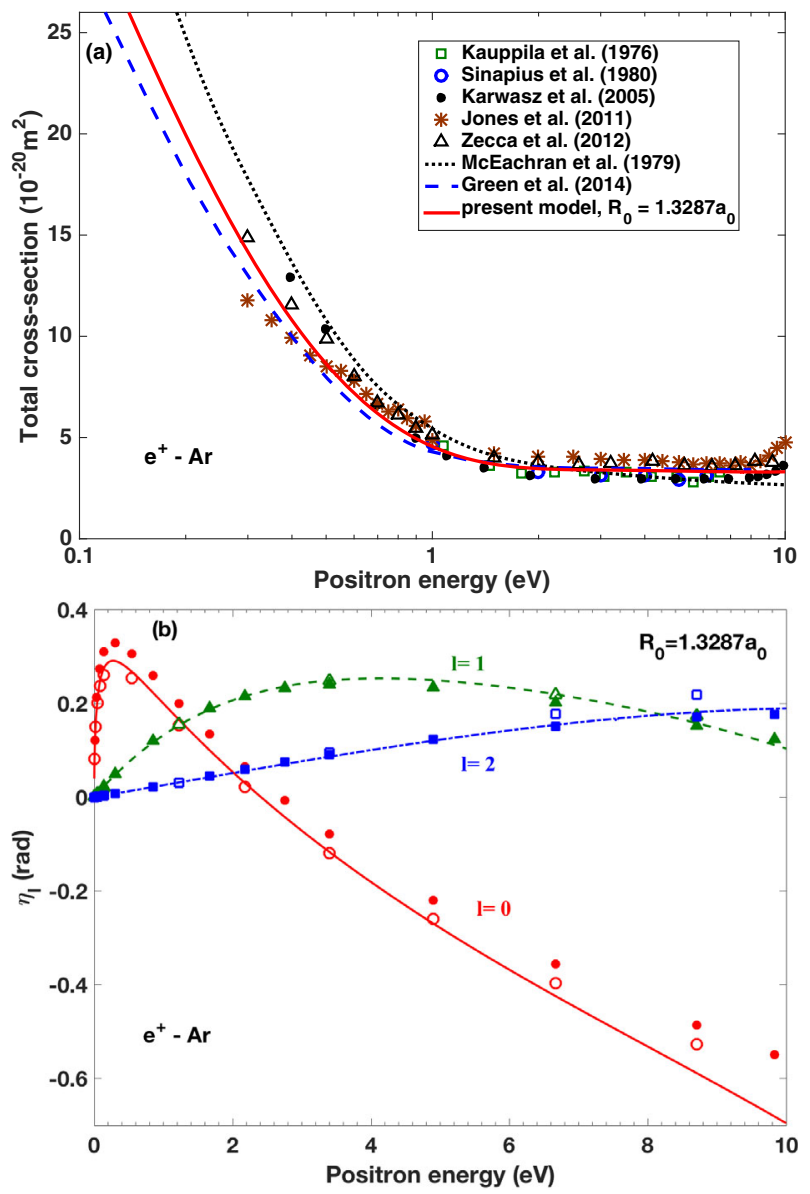

Fig. 4. (a) Elastic scattering cross section for argon. Present calculations are compared with chosen theoretical and experimental results. Theories: polarized orbital by McEachran et al. [17] and many-body theory by Green et al. [19]. Experiments: Kauppila et al. [57], Sinapius et al. [54], Karwasz et al. [51], Jones et al. [55], Zecca et al. [58]. (b) Present $s-, p$ - and $d$-wave scattering phase-shifts $\left(\eta_{l}\right)$ compared with McEachran et al. [17] (filled symbols) and Green et al. [19] (empty symbols).

\subsection{Krypton}

Figure 5 shows the results of present calculations of elastic scattering cross-sections and corresponding scattering phase-shifts for positron collision with krypton using two values of rigid sphere radius: $R_{0}=1.4751 a_{0}$ and $R_{0}=1.4977 a_{0}$. The smaller value yields cross-setcions that are compatible with the most recent experimental results by Zecca et al. [60], particularly at low energies. The higher value of $R_{0}$ give results (cross-sections and phaseshifts) being in good agreement with polarized-orbital [18] and many-body theory [19].

\subsection{Xenon}

The present calculations for xenon are shown in Figure 6. Since there are noticeable discrepancies between experimental TCSs and even more significant in theoretical models, here we present the results for two radii of rigid sphere, $R_{0}=1.7239 a_{0}$ and $R_{0}=1.687 a_{0}$.
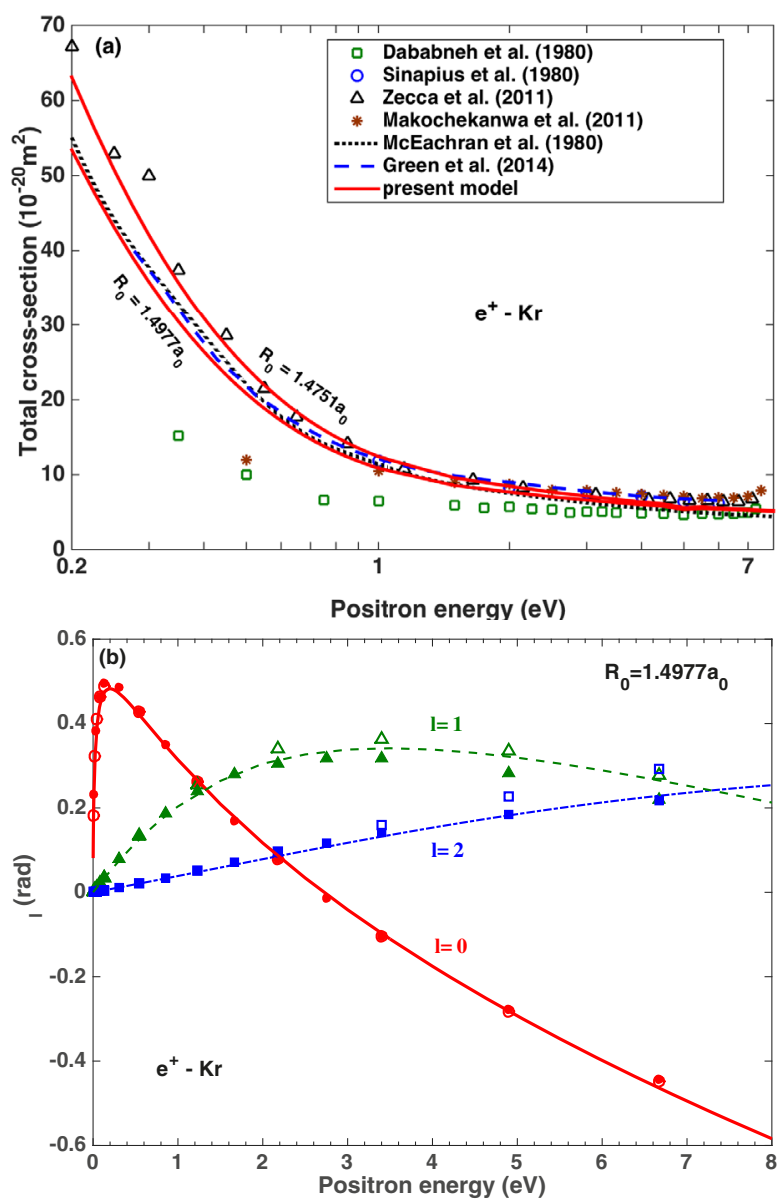

Fig. 5. Elastic scattering cross section for krypton. Present calculations for two different rigid sphere radii $\left(R_{0}\right)$ are compared with chosen theoretical and experimental results. Theories: polarized orbital by McEachran et al. [18] and many-body theory by Green et al. [19]. Experiments: Dababneh et al. [59], Sinapius et al. [54], Zecca et al. [60] and Makochekanwa et al. [61]. (b) Present $s-, p$ - and $d$-wave scattering phase-shifts $\left(\eta_{l}\right)$ compared with McEachran et al. [18] (filled symbols) and Green et al. [19] (empty symbols).

Both calculations agree with experimental data at the same level of accuracy as more advance theoretical results. Interestingly, the longer radius gives cross-sections compatible with polarized-orbital calculations by McEachran et al. [18] in almost entire considered energy range. The use of lower radius, $R_{0}=1.687 a_{0}$, gives results that follows very closely CCC calculations by Fursa and Bray [20] at very low impact energies from $0 \mathrm{eV}$ up to $0.5 \mathrm{eV}$. The many-body theory by Green et al. [19] can be also reproduced at very low energies using $R_{0}=1.6962 a_{0}$ (not shown in Fig. 6). At higher enrgies, just below the positronium formation threshold, both latter theories yields cross-sections that are much higher than all available experimental data.

\subsection{Molecular targets}

For molecular targets, in addition to spherical part of dipole polarizability, one should take into account 

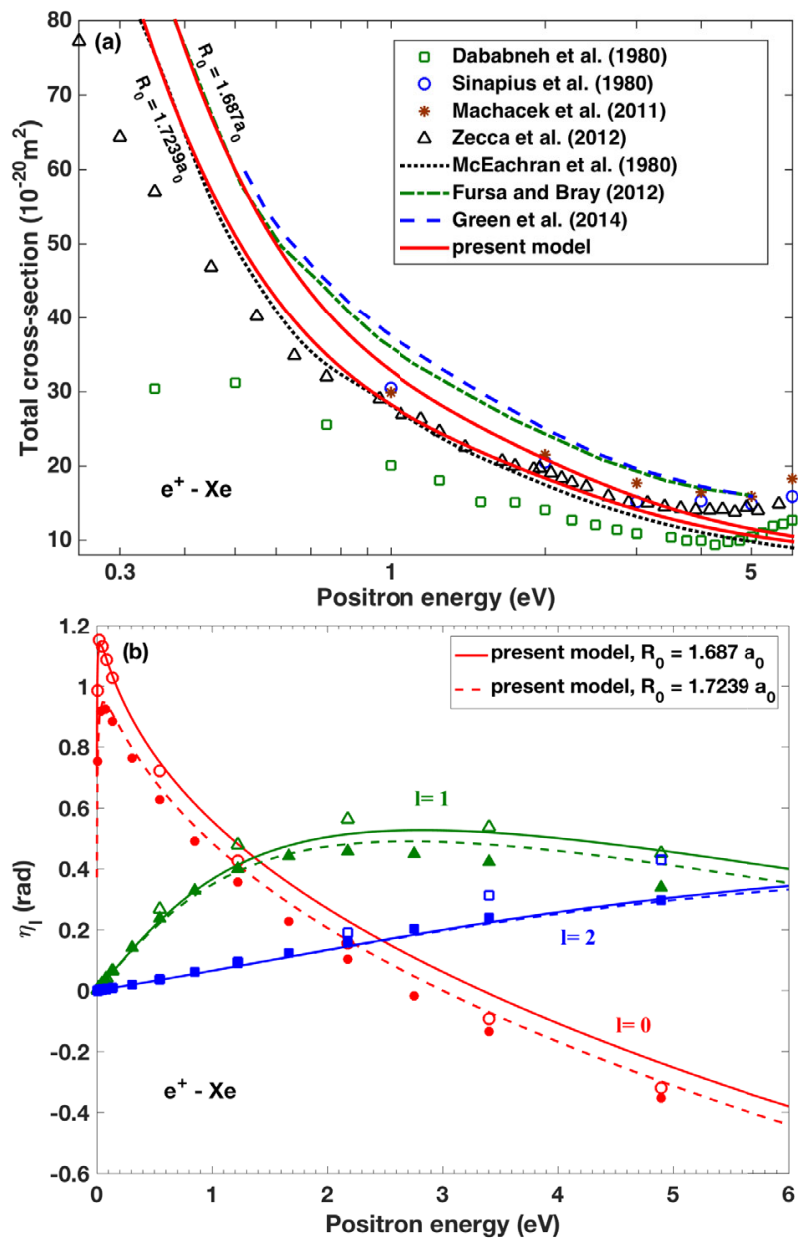

Fig. 6. Elastic scattering cross section for xenon. Present calculations for two different rigid sphere radii $\left(R_{0}\right)$ are compared with chosen theoretical and experimental results. Theories: polarized orbital by McEachran et al. [18], many-body theory by Green et al. [19] and convergent close-coupling method by Fursa and Bray [20]. Experiments: Dababneh et al. [59], Sinapius et al. [54], Machacek et al. [62], and Zecca et al. [63]. (b) Present $s$-, $p$ - and $d$-wave scattering phase-shifts $\left(\eta_{l}\right)$ compared with McEachran et al. [18] (filled symbols) and Green et al. [19] (empty symbols).

non-spherical dipole and quadrupole polarizabilities in long-range polarization potential. Here we neglect these contributions. Note also that inelastic scattering processes such as rotational and vibrational excitations can contribute to experimental total scattering cross-sections measured below the positronium formation thresholds.

Figure 7 a shows present calculations of elastic crosssections for molecular hydrogen $\left(\mathrm{H}_{2}\right)$ using three different values of rigid sphere radius: $R_{0}=0.9221 a_{0}, R_{0}=$ $0.9509 a_{0}$ and $R_{0}=0.9912 a_{0}$. Present results are compared with three the most recent experimental TCSs $[51,73,74]$ and selectively chosen recent theoretical data. Calculations with the smallest $R_{0}$ yield elastic cross-sections, which agree pretty well with experimental TCS measured by ANU group [74]. The agreement is particularly good above $2 \mathrm{eV}$, at the "flat" part of cross-sections, just below positronium formation threshold. To best
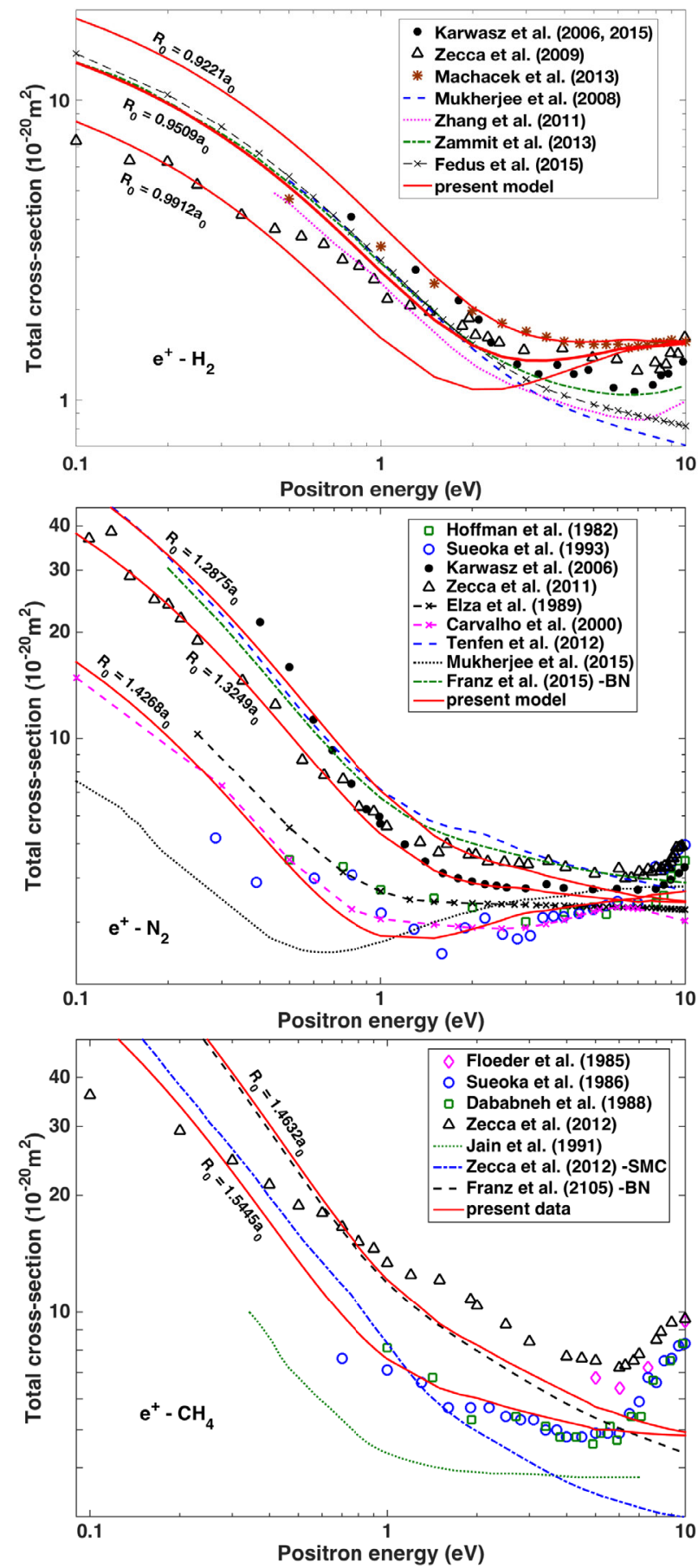

Fig. 7. Elastic scattering cross section for molecular hydrogen $\left(\mathrm{H}_{2}\right)$, nitrogen $\left(\mathrm{N}_{2}\right)$ and methane $\left(\mathrm{CH}_{4}\right)$. Present calculations for different rigid sphere radii $\left(R_{0}\right)$ are compared with chosen theoretical and experimental results. Theories for $\mathrm{H}_{2}$ : Mukherjee and Sarkar [64], Zhang et al. [65], Zammit et al. [66] and Fedus et al. [3]; theories for $\mathrm{N}_{2}$ : Elza et al. [67], Carvalho et al. [68], Tenfen et al. [69], Mukherjee and Mukherjee [70] and Franz et al. [10]; theories for $\mathrm{CH}_{4}$ : Jain and Gianturco [71], Zecca et al. [72] and Franz et al. [10]. Experiments for $\mathrm{H}_{2}$ : Fedus et al. [3], Karwasz et al. [51], Zecca et al. [73] and Machacek et al. [74]; experiments for $\mathrm{N}_{2}$ : Hoffman et al. [75], Sueoka and Hamada [76], Karwasz et al. [51] and Zecca et al. [77]; experiments for $\mathrm{CH}_{4}$ : Floeder et al. [78], Sueoka and Mori [79], Dababneh et al. [80] and Zecca et al. [72]. 
of our knowledge, so far no theoretical approach was able to reproduce this part of ANU TCS, which is the highest among available experimental datasets. However at lower energies, $E<2 \mathrm{eV}$, the smallest $R_{0}$ yields results that are higher than other experiments and theories. On the other hand, the use of the second value of $R_{0}=0.9505 a_{0}$ gives cross-sections that are consistent with ro-vibrational laboratory-frame close coupling method by Mukherjee and Sarkar [64], CCC method of Zammit et al. [66] and density functional calculations (DFT) by Fedus et al. [3] below $2 \mathrm{eV}$, giving the value of the scattering length (see next subsection) comparable with prediction of Zhang et al. [81], Zhang and Mitroy [82], Zhang et al. [83] obtain with stochastic variational method. Above $2 \mathrm{eV}$ presently calculated cross-sections agree pretty well with other experiments.

The results obtained with the largest value of $R_{0}=$ $0.9912 a_{0}$ is included in Figure 7 a mainly to show that it is possible to get results that are consistent at very low impact energies $(<0.4 \mathrm{eV})$ with TCS by Zecca et al. [73], though this experimental dataset seems to be significantly underestimated at low energies as discussed by Zhang and Mitroy [81].

Figure $7 \mathrm{~b}$ shows present calculations of elastic crosssections for molecular nitrogen $\left(\mathrm{N}_{2}\right)$ using three different values of rigid sphere radius: $R_{0}=1.2875 a_{0}, R_{0}=$ $1.3249 a_{0}$ and $R_{0}=1.4268 a_{0}$. The use of the smallest $R_{0}$ yields cross-sections consistent with ab initio calculations of Tenfen et al. [69] and DFT results obtained using Boronski-Nieminen functional by Franz et al. [10], while intermediate $R_{0}$ gives better agreement with experimental data by Zecca et al. [77] at low energies $(<1 \mathrm{eV})$ and Karwasz et al. [51] at higher energies. On the other hand, the highest value of $R_{0}$ provides results that agree with old experimental data by Sueoka and Hamada [76] and with Schwinger multichannel calculation by Carvalho et al. [68] reproducing this experimental TCS.

Figure $7 \mathrm{c}$ shows calculations of elastic cross-sections for methane $\left(\mathrm{CH}_{4}\right)$ using two different values of rigid sphere radius: $R_{0}=1.4632 a_{0}$ and $R_{0}=1.5445 a_{0}$. The use of smaller $R_{0}$ yields cross-sections consistent with DFT results using Boronski-Nieminen functional by Franz et al. [10], while larger $R_{0}$ gives better agreement with old experimental datasets by Sueoka and Mori [79] and Dababneh et al. [80]. On the other hand it is impossible with present approach to find $R_{0}$ allowing to reproduce TCS by Zecca et al. [72]. Interestingly, a slight decrease of larger $R_{0}$ value allows to follow very closely, at low energies $(<1 \mathrm{eV})$, the Schwinger multichannel calculations (SMC) reported in the same paper of Zecca et al. [72].

\subsection{Scattering lengths}

The $s$-wave scattering length for tested potential model is given analytically (in atomic units) [84]:

$$
l_{s c}=\sqrt{\alpha} \cot \left(\sqrt{\alpha} R_{0}^{-1}\right) .
$$

This analytical expression has been used to verify the correctness of present numerical model. The calculations of cross-sections were performed close to $0 \mathrm{eV}$ (from 0.00001 to $0.000001 \mathrm{eV}$ ) where the energy-dependence becomes flat. This flat cross-section value was taken as cross-section at $0 \mathrm{eV}$ to estimate the scattering length using relation $\sigma=4 \pi l_{s c}^{2}$. Both analytical (Eq. (11)) and numerical results are in perfect agreement.

Scattering lengths for all studied targets corresponding to different values of $R_{0}$ (see Tab. 1 ) are shown in Table 2 where they are compared with other determinations. Note that the increase of rigid sphere radius is accompanied by a decrease in the absolute value of the scattering length for all targets. From He to Kr different literature data for scattering lengths are quite consistent with each other and presently derived $l_{s c}$ are also comparable with them. Generally, we checked that it is often possible to adjust $R_{0}$ in such a way in order to get good agreement at low energies with cross-sectional results of most available theories and so to reproduce their estimations of $l_{s c}$.

On the contrary to lighter atoms, there are significant discrepancies in value of $l_{s c}$ reported for Xe. For example, polarized orbital calculations by McEachran and Stauffer [13] and McEachran et al. [14-18], many-body theory by Green et al. [19] and CCC results of Fursa and Bray [20] predict completely different values. This refletcs a high sensitivity of scattering calculations in the lowenergy region for xenon to the representation of the shortrange correlation potential [19]. Nevertheless, as describe in Section 3.5, using present approach it is possible to get results compatible with all these three theories at very low (near-to-zero) impact energies (in the case of polarized orbital calculations [13-18] even in the entire energy range below positronium formation threshold) by slightly adjusting the size of hard sphere.

For hydrogen, the present approach with $R_{0} \approx 0.95 a_{0}$ gives values of $l_{s c}$ that are compatible with literature data. In particular the model is consistent with the predictions by Zhang et al. [81], Zhang and Mitroy [82], Zhang et al. [83] while preserving at the same time very good agreement with experimental cross-sections at higher energies. The $l_{s c}$ values of Zhang et al. have to be considered as the most reliable at this moment since their stochastic variational approach was able to resolve a long-standing discrepancy between theoretical and experimental cross sections for positron annihilation with the $\mathrm{H}_{2}$ molecule (see Ref. [81] for more details). For other two molecules, $\mathrm{N}_{2}$ and $\mathrm{CH}_{4}$, there are much less reported values of scattering lengths, so the comparison is not so informative. Noteworthy is the fact that our simple model is compatible with cross-sections calculated by some other theories at low energies $(<1 \mathrm{eV})$ (see Fig. 7). So the present approach applied with proper rigid sphere radii should yield similar scattering lengths as those theories.

\section{Discussion and conclusions}

This work shows that very simple toy-model of interaction potential: a rigid sphere combined with an adiabatic dipole polarization $\left(\sim r^{-4}\right)$, can be used to describe positron elastic scattering by noble gases over relatively wide energy ranges below the positronium formation threshold. In this way we proved an old statement formulated 
Table 2. Positron scattering lengths $l_{s c}$ in atomic units for noble-gas atoms, molecular hydrogen, nitrogen and methane. The order appearance of scattering lengths in column 2 corresponds to the order of appearance of rigid sphere radii $\left(R_{0}\right)$ in column 3 of Table 1.

\begin{tabular}{clccc}
\hline Target & \multicolumn{1}{c}{ Present results for different $R_{0}$} & Other determinations \\
\hline $\mathrm{He}$ & -0.5976 & -0.5382 & -0.4421 & $-0.435[19] ;-0.53[14,15] ;-0.45[21]$ \\
$\mathrm{Ne}$ & & -0.6010 & $-0.467[19] ;-0.61[16] ;-0.57[21] ;-0.53[20]$ \\
$\mathrm{Ar}$ & & -4.6991 & $-4.41[19] ;-5.30[17] ;-5.05[21] ;-4.30[20] ;-4.9 \pm 0.7[58]$ \\
$\mathrm{Kr}$ & -10.9757 & -9.7120 & $-9.71[19] ;-10.4[18] ;-9.88[21] ;-11.20[20] ;-10.3 \pm 1.5[60]$ \\
$\mathrm{Xe}$ & -116.0581 & -46.7572 & $-84.50[19] ;-45[18] ;-117[20] ;-99.2 \pm 18.4[63]$ \\
$\mathrm{H}_{2}$ & -3.0856 & -2.6424 & -2.1686 & $-2.63[81] ;-2.71[82] ;-2.79[83] ;-2.49[66]$ \\
$\mathrm{N}_{2}$ & -6.1727 & -5.2125 & -3.5691 & $-2.65[85] ;-2.53[68],-9.27[24]$ \\
$\mathrm{CH}_{4}$ & -10.6753 & -7.2662 & $-13.0[86] ;-7.40[72] ;-5.65$ to $-8.50[25]$ \\
\hline
\end{tabular}

by Schrader [23] saying that any semiempirical model with disposable parameters can used to approximate positron atom short-range interactions if correct long-range behavior is included. Present model needs only one adjustable parameter - the radius of the rigid sphere, which is found to be compatible with the positions of principal maxima in the radial distribution of outermost atomic orbitals. The requirement for only one adjustable parameter is an advantage when compare to modified effective range theory (MERT) [25-29] where four to six empirical parameters are needed in order to describe variations of elastic scattering cross versus impact energy.

In more details, when compared with available experimental and theoretical data for the lightest atom - helium, the model agrees with majority of experiments and theories only at very low impact energies $(<1 \mathrm{eV})$. Probably the dipole polarizability of this target is too weak to approximate a correlation of two electrons with one positron by a simple adiabatic potential in the entire energy range below the threshold for first inelastic channel. Nevertheless two experimental datasets for He, Karwasz et al. [42-45] and Nagumo et al. [48,49], are quite consistent with the present approach over wider energy ranges for a certain value of rigid sphere radius. Interestingly, the present model is also able to accurately reproduce some of theories at very low impact energies $(E<1 \mathrm{eV})$ by adjusting the rigid sphere radius. This observation remains valid for other atomic and molecular targets.

The agreement with experiments and more advance calculations is better for neon in relatively wider energy range than for helium, but once again present model still seems to overestimate cross-sections at high energies. Nevertheless further increase of polarizability significantly improves the agreement with experiments and other theories over wider energy ranges as it is proved for heavier atoms. In particular the model constitutes a very good approximation for positron collisions with argon and krypton over almost entire energy range below the positronium formation threshold. It looks like the model works also pretty well for xenon which is characterized by the highest polarizability, though in this case the discrepancy between different experiments and theories is larger than for other atoms hindering accurate comparative analysis.

In addition the present work shows that the introduced model has a potential to describe positron elastic scattering by small molecular targets such as hydrogen, nitrogen and methane. Due to high divergence of different experimental and theoretical cross-sectional data for these molecules it is difficult to judge the reliability of present approximation. Nevertheless, as shown in this paper, it is often possible to choose radius of rigid sphere allowing to reproduce some literature data, particulraly at low energies. Present model is especially promising for describing elastic collisions with $\mathrm{H}_{2}$ and $\mathrm{N}_{2}$ in almost entire energy range below the positronium channel. Note that integral elastic scattering cross-sections for both latter molecules resemble in shapes data for $\mathrm{Ar}$ and $\mathrm{Kr}$.

Many sophisticated theories show that the dipole term alone $\left(\sim r^{-4}\right)$ is not sufficient to describe long-range part of the positron - atom interaction [1] in the entire energy range below the positronium formation threshold. For example, many-body theory [19] requires an inclusion of virtual positronium, convergent-close-coupling formalism takes into account coupling effects to virtual positronium formation channels [20], whereas polarized-orbital [13-18], DFT $[10,12]$ and model-potential $[21,22]$ approaches need higher multipole terms (quadrupole, octupole, hyperpolarizability) in long-range polarization in order to get good agreement with experiments. The present results show that the lack of attractive virtual effects, short-range correlations and higher long-range multipoles can be effectively compensated by very deep minimum of dipole polarization potential (for example $-\alpha / 2 R_{0}^{4} \approx-49 \mathrm{eV}$ for Ar, see Fig. 1). In other words such strong dipole potential is necessary to mimic attractive part of interaction at low impact energies, typically below $1 \mathrm{eV}$, when using the model of infinitive barrier for static repulsion. Note that for $\mathrm{Ar}$ and $\mathrm{Kr}$ (maybe for $\mathrm{H}_{2}$ and $\mathrm{N}_{2}$ ) this approximation is valid almost up to the positronium formation.

Furthermore, this work shows that small changes in the hard core radius allow to obatin good agreement of present results with most different theories at low energies $(E<1 \mathrm{eV})$. Saying it differently, the shift in the position of infinite barrier gives the same effect as using completly different representations of short-rage part of attractive correlation potential for impact energies at which the longrange polarization interaction $\left(\sim r^{-4}\right)$ is clearly dominant. This observation togehther with succesful applicability of MERT analysis [3,24-29] to the low-energy positron scattering suggests that contribution of short-range correlations become less important with impact energy approaching zero. 
Moreover, the present results show that the infinite rectangular barrier approximates quite well the positron - atom static repulsion in the low energy limit $(E<1 \mathrm{eV})$. Interestingly, the values of the positron scattering lengths for noble gas atoms in the static field approximation (i.e., with no polarization or other correlations included) are $0.421,0.767,1.346,1.586$ and $1.901 a_{0}$, for He to Xe, respectively. These values were calculated using DFT approach as described in reference [10] with electron density calculated on Hartree-Fock level including relativistic effects obtained with the Douglas-Kroll-Hess method. These scattering lengths are compatible with the rigid sphere radii found in this work (with the best agreement for $\mathrm{Ar}$ ).

Finally, the author is perfectly aware of a limited validity of the present toy model. In real scattering the repulsive potential is not so steep as the rectangular wall, the polarization potential needs some "cut-off" modifications $[10,12,22]$ and at the intermediate distances (some $\left.1-3 a_{0}\right)$ a sharp matching from our model is "smoothedout" by the short-range correlation potential (see Fig. 1 where the present potential is compared with other, more realistic models). The main goal of present result is to show that matching point between the repulsive and polarization potentials scales with expected "atomic dimensions". Moreover, within the frame of present approach, which is based on analytical solutions for $r^{-4}$ potential, different toy-models for the short-range correlation potential can be tested around the position of potential's minimum in order to gain better intuitive understanding of short-range interaction between low-energy positron and atomic targets.

Recent theories that go beyond "potentials" introduced by Green et al. [19], Fursa and Bray [20] and Zammit et al. [66] show that virtual effects (virtual positronium or virtual excitations, respectively) must be included to model elastic cross sections. Both approaches use the idea of temporary formation of positronium in the elastic scattering channel as postulated by Mott and Massey [87]. There are also two experimental hints (though not sufficiently clear) that the interaction of positrons at the distance of the outermost electrons is not a hard-sphere (pure elastic collisions) but rather a sticky-ball (elastic collisions + virtual attachment): (i) the evidence of enhanced annihilation signal in Ne below the positronium-formation threshold in the experiment of Szluinska and Laricchia [88] and somewhat confusing discussion by Karwasz on existence of virtual positronium in $e^{+}-$He collision [42-45]. The present theoretical approach gives space for modelling these virtual effects in semi-empirical way. Without any doubt, further non-trivial approaches are needed to understand positronatom and molecule elastic scattering.

This work is supported by the grant 2014/15/D/ST2/02358 of National Science Center in Poland. Author would like thank prof. G.P. Karwasz and Prof. Z. Idziaszek for fruitful discussions and valuable remarks. The author is indebted to Dr Jan Franz from Gdansk Technical University (Poland) who performed calculations of positron scattering lengths in static field approximations.

\section{References}

1. C.M. Surko, G.F. Gribakin, S.J. Buckman, J. Phys. B 38, R57 (2005)

2. L. Chiari, A. Zecca, Eur. Phys. J. D 68, 297 (2014)

3. K. Fedus, J. Franz, G.P. Karwasz, Phys. Rev. A 91, 062701 (2015)

4. T. Mukherjee, M. Mukherjee, Phys. Rev. A 91, 062706 (2015)

5. A. Zecca, L. Chiari, E. Trainotti, A. Sarkar, d'A.S. Sanchez, M.H.F. Bettega, M.T. do N. Varella, M.A.P. Lima, M.J. Brunger, Phys. Rev. A 85, 012707 (2012)

6. K.T. Mazon, W. Tenfen, S.E. Michelin, F. Arretche, M.-T. Lee, M.M. Fujimoto, Phys. Rev. A 82, 032704 (2010)

7. G.P. Karwasz, Eur. Phys. J. D 35, 267 (2005)

8. G.P. Karwasz, Eur. Phys. J. D 27, 53 (2006)

9. G.P. Karwasz, 13th General Conference of the European Physical Society, Bern Switzerland, 11-15 July 2005, BB10-TUE, Europhysics Conference Abstracts, Vol. 29D, p. 106

10. J. Franz, K. Fedus, G.P. Karwasz, Eur. Phys. J. D 70, 155 (2016)

11. G.P. Karwasz, R.S. Brusa, D. Pliszka, Z. Idziaszek, H. Nowakowska, Act. Phys. Pol. A 110, 605 (2006)

12. F.A. Gianturco, A. Jain, J.A. Rodriguez-Ruiz, Phys. Rev. A 48, 4321 (1993)

13. P. McEachran, A.D. Stauffer, J. Phys. B 42, 075202 (2009)

14. R.P. McEachran, D.L. Morgan, A.G. Ryman, A.D. Stauffer, J. Phys. B 10, 663 (1977)

15. R.P. McEachran, D.L. Morgan, A.G. Ryman, A.D. Stauffer, J. Phys. B 11, 951 (1978)

16. R.P. McEachran, A.G. Ryman, A.D. Stauffer, J. Phys. B 11, 551 (1978)

17. R.P. McEachran, A.G. Ryman, A.D. Stauffer, J. Phys. B 12, 1031 (1979)

18. R.P. McEachran, A.D. Stauffer, L.E.M. Campbell, J. Phys. B 13, 1281 (1980)

19. D.G. Green, J.A. Ludlow, G.F. Gribakin, Phys. Rev. A 90, $032712(2014)$

20. D.V. Fursa, I. Bray, New J. Phys. 14, 035002 (2012)

21. D. Assafrão, H.R.J. Walters, F. Arretche, A. Dutra, J.R. Mohallem, Phys. Rev. A 84, 022713 (2011)

22. L.A. Poveda, A. Dutra, J.R. Mohallem, D. Assafrão, Phys. Rev. A 87, 052702 (2013)

23. D.M. Schrader, Phys. Rev. A 20, 918 (1979)

24. Z. Idziaszek, G.P. Karwasz, Phys. Rev. A 73, 064701 (2006)

25. K. Fedus, G.P. Karwasz, Z. Idziaszek, Phys. Rev. A 88, 012704 (2013)

26. K. Fedus, Braz. J. Phys. 44, 622 (2014)

27. K. Fedus, Phys. Scr. 89, 105401 (2014)

28. K. Fedus, Braz. J. Phys. 46, 1 (2016)

29. K. Fedus, G. Karwasz, Eur. Phys. J. D 68, 93 (2014)

30. T.N. Olney, N.M. Cann, G. Cooper, C.E. Brion, Chem. Phys. 223, 59 (1997)

31. J.T. Waber, D.T. Cromer, J. Chem. Phys. 42, 4116 (1965)

32. S. Fraga, J. Karwowski, K.M.S. Saxena, Handbook of Atomic Data (Elsevier Scientific Publishing Company, Amsterdam, 1976)

33. D.C. Ghosh, R. Biswas, Int. J. Mol. Sci. 3, 87 (2002)

34. J.B. Mann, Atomic Structure Calculations II. Hartree-Fock wave functions and radial expectation values: hydrogen to lawrencium, LA-3691 (Los Alamos Scientific Laboratory, USA, 1968) 
35. M.A. Spackman, E.N. Maslen, J. Phys. Chem. 90, 2020 (1986)

36. B. Cordero, V. Gómez, A.E. Platero-Prats, M. Revés, J. Echeverría, E. Cremades, F. Barragán, S. Alvarez, in Covalent radii revisited, Dalton Trans. 21, 2832 (2008)

37. A.M. James, M.P. Lord, Macmillan's Chemical and Physical Data (Macmillan, London, UK, 1992)

38. P. Pyykkö, S. Riedel, M. Patzschke, Chem. Eur. J. 11, $3511(2005)$

39. J. McMurry, Fundamentals of organic chemistry, 7th edn. (Cornell University, 2011), p. 11

40. T.S. Stein, W.E. Kauppila, V. Pol, J.H. Smart, G. Jesion, Phys. Rev. A 17, 1600 (1978)

41. T. Mizogawa, Y. Nakayama, T. Kawaratani, M. Tosaki, Phys. Rev. A 31, 2171 (1985)

42. G.P. Karwasz, D. Pliszka, A. Zecca, R.S. Brusa, Nucl Instrum. Methods Phys. Res. Sect. B 240, 666 (2005)

43. G.P. Karwasz, D. Pliszka, A. Zecca, R.S. Brusa, Nucl Instrum. Methods Phys. Res. Sect. B 251, 310 (2006)

44. G.P. Karwasz, D. Pliszka, A. Zecca, R.S. Brusa, Nucl. Instrum. Methods Phys. Res. Sect. B 251, 517 (2006)

45. G.P. Karwasz, D. Pliszka, A. Zecca, R.S. Brusa, Nucl. Instrum. Methods Phys. Res. Sect. B 251, 520 (2006)

46. J.P. Sullivan, C. Makochekanwa, A. Jones, P. Caradonna, S.J. Buckman, J. Phys. B 41, 081001 (2008)

47. P.M. Jay, P.G. Coleman, Phys. Rev. A 82, 012701 (2010)

48. K. Nagumo, Y. Nitta, M. Hoshino, H. Tanaka, Y. Nagashima, J. Phys. Soc. Jpn 80, 064301 (2011)

49. K. Nagumo, Y. Nitta, M. Hoshino, H. Tanaka, Y. Nagashima, J. Phys. Soc. Jpn 80, 098001 (2011)

50. S.E. Fayer, A. Loreti, S.L. Andersen, Á. Kövér, G. Laricchia, J. Phys. B 49, 075202 (2016)

51. G.P. Karwasz, D. Pliszka, R.S. Brusa, Nucl. Instrum. Methods B 247, 68 (2006)

52. J.P. Sullivan, C. Makochekanwa, A. Jones, P. Caradonna, D.S. Slaughter, J. Machacek, R.P. McEachran, D.W. Mueller, S.J. Buckman, J. Phys. B 44, 035201 (2011)

53. H. Wu, I. Bray, D.V. Fursa, A.T. Stelbovics, J. Phys. B 37, L1 (2004)

54. G. Sinapius, W. Raith, W.G. Wilson, J. Phys. B 13, 4079 (1980)

55. A.C.L. Jones, C. Makochekanwa, P. Caradonna, D.S. Slaughter, J.R. Machachek, R.P. McEachran, J.P. Sullivan, S.J. Buckman, A.D. Stauffer, I. Bray, D.V. Fursa, Phys. Rev. A 83, 032701 (2011)

56. K. Nagumo, Y. Nitta, M. Hoshino, H. Tanaka, Y. Nagashima, Eur. Phys. J. D 66, 81 (2012)

57. W.E. Kauppila, T.S. Stein, G. Jesion, Phys. Rev. Lett. 36, $580(1976)$

58. A. Zecca, L. Chiari, E. Trainotti, D.V. Fursa, I. Bray, A. Sarkar, S. Chattopadhyay, K. Ratnavelu, M.J. Brunger, J. Phys. B 45, 015203 (2012)

59. M.S. Dababneh, W.E. Kauppila, J.P. Downing, F. Laperriere, V. Pol, J.H. Smart, T.S. Stein, Phys. Rev. A 22, 1872 (1980)

60. A. Zecca, L. Chiari, E. Trainotti, D.V. Fursa, I. Bray, M.J. Brunger, Eur. Phys. J. D 64, 317 (2011)

61. C. Makochekanwa, J.R. Machacek, A.C.L. Jones, P. Caradonna, D.S. Slaughter, R.P. McEachran, J.P. Sullivan, S.J. Buckman, S. Bellm, B. Lohmann, D.V. Fursa, I. Bray, D.W. Mueller, A.D. Stauffer, M. Hoshino, Phys. Rev. A 83, $032721(2011)$
62. J.R. Machacek, C. Makochekanwa, A.C.L. Jones, P. Caradonna, D.S. Slaughter, R.P. McEachran, J.P. Sullivan, S.J. Buckman, S. Bellm, B. Lohmann, D.V. Fursa, I. Bray, D.W. Mueller, A.D. Stauffer, New J. Phys. 13, 125004 (2011)

63. A, Zecca, L. Chiari, E. Trainotti, M.J. Brunger, J. Phys. B 45, 085203 (2012)

64. T. Mukherjee, N.K. Sarkar, J. Phys. B 41, 125201 (2008)

65. R. Zhang, K.L. Baluja, J. Franz, J. Tennyson, J. Phys. B 44, 035203 (2011)

66. M.C. Zammit, D.V. Fursa, I. Bray, Phys. Rev. A 87, 020701(R) (2013)

67. B.K. Elza, T.L. Gibson, M.A. Morrison, B.C. Saha, J. Phys. B 22, 113 (1989)

68. R.C. Carvalho, M.T. do N. Varella, M.A.P. Lima, E.P. Silva, J.S.E. Germano, Nucl. Instrum. Methods Phys. Res. Sect. B 171, 33 (2000)

69. W. Tenfen, K.T. Mazon, S.E. Michelin, F. Arretche, Phys. Rev. A 86, 042706 (2012)

70. T. Mukherjee, M. Mukherjee, Phys. Rev. A 91, 062706 (2015)

71. A. Jain, F.A. Gianturco, J. Phys. B 24, 2387 (1991)

72. A. Zecca, L. Chiari, E. Trainotti, A. Sarkar, d'A.S. Sanchez, M.H.F. Bettega, M.T. do N. Varella, M.A.P. Lima, M.J. Brunger, Phys. Rev. A 85, 012707 (2012)

73. A. Zecca, L. Chiari, A. Sarkar, K.L. Nixon, M.J. Brunger. Phys. Rev. A 80, 032702 (2009)

74. J.R. Machacek, E.K. Anderson, C. Makochekanwa, S.J. Buckman, J.P. Sullivan, Phys. Rev. A 88, 042715 (2013)

75. K.R. Hoffman, M.S. Dababneh, Y.F. Hsieh, W.E. Kauppila, V. Pol, J.H. Smart, T.S. Stein, Phys. Rev. A 25, $1393(1982)$

76. O. Sueoka, A. Hamada, J. Phys. Soc. Jpn 62, 2669 (1993)

77. A. Zecca, L. Chiari, A. Sarkar, M.J. Brunger New J. Phys. 13, 115001 (2011)

78. K. Floeder, D. Fromme, W. Raith, A. Schwab, G. Sinapius, J. Phys. B 18, 3347 (1985)

79. O. Sueoka, S. Mori, J. Phys. B 19, 4035 (1986)

80. M.S. Dababneh, Y.F. Hsieh, W.E. Kauppila, C.K. Kwan, S.J. Smith, T.S. Stein, M.N. Uddin, Phys. Rev. A 38, 1207 (1988)

81. J.-Y. Zhang, J. Mitroy, K. Varga, Phys. Rev. Lett. 103, $223202(2009)$

82. J.-Y. Zhang, J. Mitroy, Phys. Rev. A 83, 022711 (2011)

83. J.-Y. Zhang, Y.-J. Yang, Y. Qian, Z.-C. Yan, U. Schwingenschlögl, Phys. Rev. A 89, 042703 (2014)

84. I.T. Iakubov, A.G. Khrapak, Phys. Rev. A 51, 5043 (1995)

85. C.J. Evans, H.L. Mansou, J. Phys. B 20, 5925 (1987)

86. Y. Frongillo, B. Plenkiewicz, J.-P. Jay-Gerin, A. Jain, Phys. Rev. E 50, 4754 (1994)

87. N.F. Mott, H.S.W. Massey, Theory of Atomic Collisions (Oxford University Press, London, 1965), pp. 550-555

88. M. Szluinska, G. Laricchia, Nucl. Instrum. Meth. B 221, $100(2004)$

Open Access This is an open access article distributed under the terms of the Creative Commons Attribution License (http://creativecommons.org/licenses/by/4.0), which permits unrestricted use, distribution, and reproduction in any medium, provided the original work is properly cited. 